\title{
Estrogen administration attenuates post-stroke depression by enhancing CREB/BDNF/TrkB signaling in the rat hippocampus
}

\author{
HUIGANG JIANG $^{1}$, LI XIAO ${ }^{2}$, KUNLIN JIN $^{3,4}$ and BEI SHAO ${ }^{3}$ \\ ${ }^{1}$ Department of Neurology, Yiwu City Center Hospital, Wenzhou Medical University, Yiwu, Zhejiang 322000; \\ ${ }^{2}$ Department of Neurology, Shaoyang City Center Hospital, Shaoyang, Hunan 422000; \\ ${ }^{3}$ Department of Neurology, First Affiliated Hospital, Wenzhou Medical University, \\ Wenzhou, Zhejiang 325000, P.R. China; ${ }^{4}$ Department of Pharmacology and Neuroscience, \\ University of North Texas Health Science Center at Fort Worth, TX 76107, USA
}

Received July 5, 2020; Accepted October 9, 2020

DOI: $10.3892 /$ etm.2021.9850

\begin{abstract}
A previous study demonstrated that $17 \beta$-estradiol (E2), which is an antidepressant, can ameliorate post-stroke depression (PSD); however, the underlying mechanisms governing this remain largely unknown. Therefore, the present study developed a PSD model in rats, which was induced by left middle cerebral artery occlusion followed by exposure to chronic mild stress for 2 weeks. The results revealed that the activity of the cAMP response element-binding protein (CREB), a cellular transcription factor, and the associated brain-derived neurotrophic factor (BDNF)/tyrosine kinase B (TrkB) signaling were all attenuated in the hippocampus in PSD rats. The depression-like behaviors were significantly improved after treatment with E2, along with increased CREB and the BDNF/TrkB signaling activity. These results provide novel insight into the molecular basis of PSD, and suggest the potential involvement of CREB/BDNF/TrkB signaling in E2-mediated improvement of PSD in rats.
\end{abstract}

\section{Introduction}

Strokes are the second leading cause of mortality worldwide (1). In China, the age-adjusted incidence of a first-time stroke is not markedly different compared with developed countries (2). Post-stroke depression (PSD) is a common occurrence following a stroke $(3,4)$, and is associated with increased disability, cognitive impairment, suicidal behavior and mortality (1). PSD has been estimated to affect $33 \%$ of patients worldwide (5-7). Although the mechanisms underlying the development of PSD

Correspondence to: Dr Bei Shao, Department of Neurology, First Affiliated Hospital, Wenzhou Medical University, Ward 361, No. 3 Hospital Building, Nanbaixiang Street, Wenzhou, Zhejiang 325000, P.R. China

E-mail: shaobei56@126.com

Key words: estrogen, stroke, depression, brain-derived neurotrophic factor, tyrosine kinase B, cAMP response element-binding protein, hippocampus remain largely unknown, a number of hypotheses, including a neurotrophic effect, have been proposed (8). Studies have documented that stress decreases the expression of brain-derived neurotrophic factor (BDNF) mRNA in the hippocampus $(9,10)$, and direct hippocampal infusions of BDNF protein can produce antidepressant effects in rodents (11). In addition, it has been demonstrated that serum concentrations of BDNF are decreased in human patients with PSD (12). BDNF may act through high-affinity interactions with its specific receptor, tyrosine kinase B (TrkB) $(13,14)$, as mice lacking TrkB have been demonstrated to exhibit increased anxiety-like behaviors (15). Notably, expression of BDNF is partly regulated by cAMP response element-binding protein (CREB), which is a cellular transcription factor (16). Dysregulation of CREB expression or activity has been associated with mood disorders, including depression (17). In addition, it has been reported that CREB and the BNDF-TrkB signaling can form a positive feedback loop $(16,18)$, CREB can be activated by TrkB signaling through phosphorylation, and phosphorylated (p)CREB will subsequently activate BDNF to enhance TrkB signaling (19). Estrogen is an effective mood regulator that has been demonstrated to induce antidepressant like effects on a depression model in rats (20). Estrogen also serves a neuroprotective role through estrogen-dependent alterations in cell survival (21), enhancement of anti-apoptotic gene expression and neurogenesis (22). A previous study demonstrated that $17 \beta$-estradiol (E2), which is an antidepressant, can ameliorate PSD. The similarities between the effects of estrogen and BDNF on hippocampal physiology and behavior have also been observed (23-25). Therefore, the present study hypothesized that $\mathrm{CREB} / \mathrm{BDNF} / \mathrm{TrkB}$ signaling may serve a role in the E2-mediated improvement of PSD in rats. In the present study, the CREB/BDNF/TrkB signaling activation in PSD rats was investigated following moderate doses of $\mathrm{E} 2$ treatment. The results of the present study may provide novel insights into the molecular basis of E2-mediated improvement of PSD.

\section{Materials and methods}

Animals. A total of 120 two-month-old female Sprague-Dawley (SD) rats, weighing between 220 and $250 \mathrm{~g}$, were obtained from the Animal Center of Wenzhou Medical University in 
China. The rats were housed four per cage and maintained on a $12 \mathrm{~h}$ light/dark cycle, with lights turned on at 7:00 a.m. and temperature at $21 \pm 2^{\circ} \mathrm{C}$; and provided with ad libitum access to food and water. The groups of ovariectomized (OVX) female SD rats used included the following: i) Sham-Operated rats treated with vehicle (control + vehicle; $n=16$ ); ii) PSD rats treated with vehicle (PSD + vehicle; $n=16$ ); iii) PSD rats treated with E2 (PSD + E2; n=16); iv) PSD rats treated with $\mathrm{K} 252 \mathrm{a}$ and $\mathrm{E} 2(\mathrm{PSD}+\mathrm{K} 252 \mathrm{a}+\mathrm{E} 2 ; \mathrm{n}=16) ; \mathrm{v})$ PSD rats treated with U0126 and E2 (PSD + U0126 + E2, n=16). PSD rats were randomly divided into matched subgroups for subsequent K252a and U0126 with vehicle treatment as follows: PSD rats treated with K252a and vehicle groups (PSD + K252a + vehicle; $n=8)$ and PSD rats treated with U0126 vehicle groups $(\mathrm{PSD}+\mathrm{U} 0126$ + vehicle; $\mathrm{n}=8)$. PSD rats were subcutaneously injected with E2 at 1,10 or $25 \mu \mathrm{g}$ ( $\mathrm{n}=8$ per group). All experiments were performed in accordance with the National Institutes of Health Guide for the Care and Use of Laboratory Animals (26), with the approval of the Institutional Animal Care and Use Committee at First Affiliated Hospital, Wenzhou Medical University, Wenzhou. The experimental paradigm is described in (Fig. 1). Process A indicates the middle cerebral artery occlusion. Process B indicates the chronic mild stress. Process $\mathrm{C}$ indicates the estrogen or oil treatment.

OVX surgery. Female SD rats were anesthetized via intraperitoneal injection with $10 \%$ chloral hydrate $(320 \mathrm{mg} / \mathrm{kg})$ and showed no obvious signs of peritonitis. All rats were subjected to an ovariectomy prior to grouping. Bilateral ovariectomy was performed according to previously published procedures (27). Briefly, a single midline incision was made in the lower abdominal area to expose the ovary, and oviducts were bilaterally ligated and the ovaries removed. After suturing the muscles and skin, the animals were returned to their cages to recover for 1 week in the same conditions as aforementioned.

Transient focal cerebral ischemia. After 1 week had passed, the OVX rats were subjected to focal ischemia as previously described $(24,28)$. Briefly, the rats were anesthetized with $10 \%$ chloral hydrate $(320 \mathrm{mg} / \mathrm{kg})$ and the left common carotid artery was exposed. The external carotid artery and its branches were subsequently isolated and coagulated. A 3-0 nylon suture with a blunted tip was inserted into the internal carotid artery through the external carotid artery stump, which advanced to the anterior cerebral artery to occlude the middle cerebral artery (MCA). After occluding the MCA for $90 \mathrm{~min}$, the suture was removed to restore blood flow, and the rats were placed in a cage under an infrared heating lamp at $35 \pm 2^{\circ} \mathrm{C}$ until they had recovered from anesthesia. Rectal temperature was maintained at $37.0 \pm 0.5^{\circ} \mathrm{C}$ using a thermostat-controlled heating pad. Sham-operated rats underwent the same surgery and recovery protocol, except that the MCA was not occluded.

Chronic mild stress. A period of 1 week post-MCAO, the animals were subjected to chronic mild stress (CMS) for 2 consecutive weeks. Each week, the stress regime included: i) Food or water deprivation for $12 \mathrm{~h}$; ii) Cage tilt $\left(45^{\circ}\right)$; iii) Overnight illumination; iv) Soiled cage $(250 \mathrm{ml}$ water in sawdust bedding); v) Swimming in $4^{\circ} \mathrm{C}$ water; and vi) Placement in a restraining device as previously described $(20,24)$. Each rat received one form of stress each day. Control animals were housed in separate rooms and did not undergo the stress regime.

\section{Behavior tests}

Open-field test. The open-field test consisted of a wooden box (100 cm in length and $100 \mathrm{~cm}$ in width) with the wall painted black (40 cm high); the non-reflective floor was divided into $25(5 \times 5)$ equal squares $(20 \times 20 \mathrm{~cm})$. Each room test was dimly illuminated with one $25 \mathrm{~W}$ red bulb located $130 \mathrm{~cm}$ above the open-field floor. The animals were placed at the center of the open field arena and tested in a quiet room. The frequency of rearing and line crossing activity of the test subject was quantified over a 5-min period by trained and experienced observers were blinded to the treatment groups. Rats crossing the line were scored when all four paws were removed from one square and entered another. Rears were scored when both front paws were raised from the floor, and climbs were scored when an animal leaned its front paws against a wall. Open field activities scores were monitored in all groups at baseline, at 2 weeks after CMS and at 2 weeks following treatment.

Sucrose preference tests. After 1 week of acclimatization to the Animal Center, rats were trained to consume $1 \%(\mathrm{w} / \mathrm{v})$ sucrose solution, as previously described (24). A period of 3 days after this, and after $23 \mathrm{~h}$ of food and water deprivation, a $1 \mathrm{~h}$ baseline test was performed, in which rats could select between two pre-weighted bottles, one with $1 \%(\mathrm{w} / \mathrm{v})$ sucrose solution and the other with tap water. The maximum percentage of body weight loss following the $23 \mathrm{~h}$ food and water deprivation was as follows: Baseline group 5.9\% vs. $2 \mathrm{~W}$ CMS group 5.5\% vs. 2W Treatment group 5.4\%.

The position of the two bottles in the $1 \mathrm{~h}$ baseline test (left/right sides of the cages) varied randomly between trials. The sucrose preference tests were performed at 2 weeks following CMS and at 2 weeks following treatment under similar conditions throughout the experiment. The relative sucrose intake $(\mathrm{g} / \mathrm{g})$ was the absolute sucrose intake per gram of rat body weight, whereas sucrose preference (SP) was calculated according to the following ratio: $\mathrm{SP}=$ sucrose intake $(\mathrm{g}) /[$ sucrose intake $(\mathrm{g})+$ water intake $(\mathrm{g})]$.

E2 administration. PSD rats were injected subcutaneously with $10 \mu \mathrm{g}$ E2 (Sigma-Aldrich; Merck KGaA) in $0.1 \mathrm{ml}$ sesame oil at 09:00 and 10:00 am for 14 consecutive days from the establishment of the PSD model. The PSD group and control group rats received $0.1 \mathrm{ml}$ sesame oil using the same protocol.

Subventricle zone (SVZ) infusions of K252a and U0126. PSD rats were anesthetized with $10 \%$ chloral hydrate $(320 \mathrm{mg} / \mathrm{kg})$ prior to stereotaxic surgery. A small burr hole was drilled in the left hemisphere of the brain and a stainless steel needle (26 gauge) was inserted into the left ventricle (coordinates $0.9 \mathrm{~mm}$ posterior from bregma, $1.5 \mathrm{~mm}$ from the midline and $4.0 \mathrm{~mm}$ from the brain surface). After fixing the guide cannulas to the skull, a substitute cannula (length, $8.5 \mathrm{~mm}$; external diameter, $0.35 \mathrm{~mm}$ ) was inserted into the guide cannula to prevent a block of tubing. The guide cannula was removed 


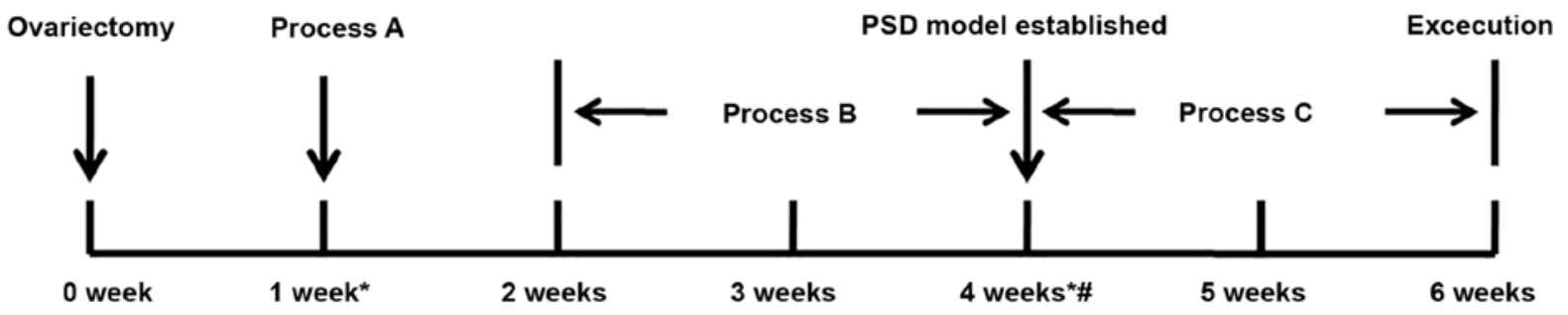

Figure 1. Experimental design. Process A indicates the middle cerebral artery occlusion (estrogen and PSD group) and sham operation. Process B indicates the chronic mild stress (estrogen and PSD group) and the common feeding service (control group). Process C indicates the estrogen treatment (estrogen group) and oil treatment (PSD and control group). "Time-point, open-field test and sucrose preference index; ${ }^{\#}$ Time-point, subventricle zone infusions of K252a and U0126. PSD, post-stroke depression.

3 days later, and the wound was disinfected. K252a, a TrkB specific blocker, was dissolved in $1 \%$ sterilized DMSO and prepared into a $40 \mathrm{mmol} / 1$ solution. Each $3 \mu \mathrm{l}$ liquid contained $120 \mathrm{nmol} \mathrm{K} 252 \mathrm{a}(10)$. K252a solution $(3 \mu \mathrm{l})$ was injected into the lateral ventricle (29). U0126 is a mitogen activated protein kinase (MAPK) blocker that inhibits CREB phosphorylation and dissolves U0126 in $1 \%$ DMSO $(0.3 \mathrm{~g} / \mu \mathrm{l}), 3 \mu \mathrm{l}$ of which was injected into the lateral ventricle of the rats in the present study $(30,31)$. Each PSD rat was randomly assigned to artificial cerebrospinal fluid (aCSF), U0126 and K252a groups. Each drug ( $3 \mu \mathrm{l}$ in total) was unilaterally injected into the lateral ventricle via an injection cannula (external diameter, $0.35 \mathrm{~mm}$ ) extending $0.5 \mathrm{~mm}$ below the tip of the guide cannula at a rate of $1 \mu \mathrm{l} / \mathrm{min}$ using a $10 \mathrm{ml}$ Hamilton syringe. Drug administration was performed continuously for 3 days. After establishing the model, rats were treated with E2 $(10 \mu \mathrm{g})$ or vehicle for 2 weeks.

Measurement of serum E2. After 2 weeks of $(1,10$ or $25 \mu \mathrm{g})$ E2 therapy, rats were anesthetized with $10 \%$ chloral hydrate at a dose of $320 \mathrm{mg} / \mathrm{kg}$, the abdominal cavity was exposed, the abdominal aorta was isolated, and $2-3 \mathrm{ml}$ of blood was extracted. Cervical dislocation was used as the method of euthanasia. No rat exhibited signs of peritonitis after the administration of $10 \%$ chloral hydrate. Rat deaths were confirmed by observing breathing and cardiac arrest. Glass tubes containing whole blood from the abdominal aorta were kept at room temperature for $1-2 \mathrm{~h}$ and then centrifuged at $4^{\circ} \mathrm{C}, 1,800 \mathrm{x}$ g for $15 \mathrm{~min}$. Serum was collected into glass vials and stored at $-80^{\circ} \mathrm{C}$. Serum levels of estradiol were sent to the Guangzhou Kingmed Diagnostics Group Co., Ltd. for testing via Enhanced Estradiol Assay kit (cat. no. CLA-4664; DRG Instruments GmbH). The ADVIA Centaur Automatic chemiluminescence immunoassay system (Siemens AG) was used to assay the serum E2 levels, according to the manufacturer's protocol. The endogenous estradiol contained in the sample was released from its binding protein using an estradiol assay kit release agent. The anti-estradiol rabbit polyclonal antibody (1:100; cat. no. CLA-4664; DRG Instruments $\mathrm{GmbH}$ ) labeled with acridine ester was then added to bind the available estradiol. Finally, estradiol derivatives were added to the reactants to capture the solid phase and bind to estradiol competitively and acridin-labeled antibodies. After washing, acids and bases are added to start the chemiluminescence reaction for $10 \mathrm{~min}$ at room temperature. All experiments were performed twice. The lower level of detectability was $3 \mathrm{pg} / \mathrm{ml}$.
Immunohistochemistry. Animals were sacrificed after 2 weeks of treatment. Rats were anesthetized with $10 \%$ chloral hydrate and decapitated. The rat brains were fixed in $4 \%$ paraformaldehyde in PBS for $24 \mathrm{~h}$ at $4^{\circ} \mathrm{C}$, and then dehydrated in a graded series of alcohol, placed in xylene until transparent and embedded in paraffin via treatment at $60^{\circ} \mathrm{C}$ oven. Brain tissues were cut to $4 \mathrm{~mm}$-thick sections using a microtome. The sections were blocked in $3 \% \mathrm{H}_{2} \mathrm{O}_{2}$ for $10 \mathrm{~min}$ at room temperature, and 3\% normal goat serum (OriGene Technologies, Inc.), and then incubated with rabbit polyclonal antibodies against phospho cAMP response element-binding protein (pCREB; 1:800; cat. no. 9198; Cell Signaling Technology, Inc.), CREB (1:3,000; cat. no. 9197; Cell Signaling Technology, Inc.), BDNF (1:200; cat. no. ab6201; Abcam) at $4{ }^{\circ} \mathrm{C}$ overnight. HRP-labeled goat anti-rabbit secondary antibody (1:1,000; cat. no. TA140003; OriGene Technologies, Inc.) for $30 \mathrm{~min}$ at $37^{\circ} \mathrm{C}$ and diaminobenzidine from the Streptavidin-Peroxidase kit (OriGene Technologies, Inc.) were used to visualize the signals. Hematoxylin was used for counterstaining for 1-2 $\mathrm{min}$ at room temperature. The controls included incubation without any primary or secondary antibodies at $37^{\circ} \mathrm{C}$ for $30 \mathrm{~min}$. Finally, the sections were observed under a NIKON fluorescence microscope (magnification, $x 40$, x200 and x400; Nikon Corporation).

Western blot analysis. The hippocampus was also dissected from rats euthanized via cervical dislocation. The tissue samples were homogenized in ice-cold lysis buffer and phenylmethanesulfonyl fluoride. The homogenates were centrifuged at $4^{\circ} \mathrm{C}$ and $21,900 \times \mathrm{g}$ for $15 \mathrm{~min}$. Protein concentrations were measured using a BCA Protein Assay Reagent kit (Beyotime Institute of Biotechnology). Protein (50 $\mu \mathrm{g}$ per lane) was loaded and separated via 12\% SDS-PAGE and transferred onto PVDF membranes (EMD Millipore). After blocking for $90 \mathrm{~min}$ at room temperature with $5 \%$ non-fat dried milk in TBS, the membranes were incubated overnight at $4^{\circ} \mathrm{C}$ with anti- $\beta$-actin (1:1,000; cat. no. ab8226; Abcam), polyclonal anti-CREB (1:5,000; cat. no. 9197; Cell Signaling Technology, Inc.), anti-phospho-CREB (1:5,000; cat. no. 9198; Cell Signaling Technology, Inc.) at Ser133, anti-BDNF (1:1,000; cat. no. ab6201; Abcam), anti-pTrkB (1:3,000; cat. no. 4168; Cell Signaling Technology, Inc.) or TrkB (1:3,000; cat. no. 4603; Cell Signaling Technology, Inc.) and shaken on a rotator at $4^{\circ} \mathrm{C}$ for overnight. The membranes were washed three times with TBS containing $0.1 \%$ Tween-20 (TBST) for $7 \mathrm{~min}$ each, and subsequently incubated in HRP-labeled goat anti-rabbit 
Table I. Open field activities scores in all groups at baseline, at 2 weeks after CMS and at 2 weeks following treatment.

\begin{tabular}{|c|c|c|c|c|c|c|}
\hline \multirow[b]{2}{*}{ Group (n) } & \multicolumn{3}{|c|}{ Crossing activity } & \multicolumn{3}{|c|}{ Rearing activity } \\
\hline & Baseline & 2W CMS & $2 \mathrm{~W}$ Treatment & Baseline & 2W CMS & $2 \mathrm{~W}$ Treatment \\
\hline CON (16) & $71.4 \pm 5.74$ & $64.5 \pm 4.47$ & $65.3 \pm 4.75$ & $10.4 \pm 1.66$ & $9.6 \pm 1.64$ & $10.1 \pm 1.24$ \\
\hline PSD (16) & $70.8 \pm 5.35$ & $36.8 \pm 3.42^{\mathrm{a}}$ & $32.7 \pm 6.15$ & $11.8 \pm 1.24$ & $7.6 \pm 1.21^{\mathrm{a}}$ & $6.3 \pm 1.02$ \\
\hline E2 (16) & $68.2 \pm 6.48$ & $36.5 \pm 3.47^{\mathrm{a}}$ & $58.6 \pm 4.45^{\mathrm{b}}$ & $10.2 \pm 2.22$ & $5.4 \pm 0.77^{\mathrm{a}}$ & $9.6 \pm 0.80^{\mathrm{b}}$ \\
\hline E2+U0126 (16) & $72.1 \pm 7.32$ & $35.6 \pm 4.17^{a}$ & $41.1 \pm 5.92^{c}$ & $13.2 \pm 1.86$ & $4.2 \pm 0.53^{\mathrm{a}}$ & $7.3 \pm 0.61^{\mathrm{c}}$ \\
\hline $\mathrm{E} 2+\mathrm{K} 252 \mathrm{a}(16)$ & $73.5 \pm 8.22$ & $40.4 \pm 5.24^{\mathrm{a}}$ & $40.2 \pm 4.37^{c}$ & $11.1 \pm 1.84$ & $5.7 \pm 0.76^{\mathrm{a}}$ & $6.9 \pm 0.52^{c}$ \\
\hline
\end{tabular}

${ }^{\mathrm{a}} \mathrm{P}<0.05$ vs. $\mathrm{CON}$; ${ }^{\mathrm{b}} \mathrm{P}<0.05$ vs. $\mathrm{PSD}$; ${ }^{\mathrm{P}}<0.05$ vs. E2. CON, control; $\mathrm{PSD}$, post-stroke depression; E2, 17 $\beta$-estradiol; W, week; CMS, chronic mild stress.

secondary antibody (1:5,000; cat. no. A0208; Beyotime Institute of Biotechnology) with TBST for $1 \mathrm{~h}$ at room temperature. Membranes were then washed three times with TBST for $10 \mathrm{~min}$ each. The resulting antigen-antibody-peroxidase complexes were detected by an ECL detection system (Beyotime Institute of Biotechnology). Densitometric analysis of the western blot images was performed using a Bio-Rad GS-710 Calibrated Imaging Densitometer and quantified using Quantity One software (Bio-Rad Laboratories, Inc; version 4.5.2).

Statistical analysis. Significant differences among groups were determined using one-way ANOVA using SPSS software (version 23.0; IBM, Corp). Post-hoc comparisons were performed using Tukey's post hoc test. All values are reported as the mean \pm standard error of the mean. $\mathrm{P}<0.05$ was considered to indicate a statistically significant difference.

\section{Results}

Successful PSD model. To establish a PSD model, rats that had undergone surgical removal of the ovaries were subjected to MCAO and 2 weeks of CMS (Fig. 1). OVX rats without MCAO and CMS were used as controls. As presented in Table I, the rats undergoing CMS displayed a significant decrease in crossing activity and rearing activity compared with control rats $(\mathrm{P}<0.05)$, and they drank sucrose significantly less compared with the control rats $(\mathrm{P}<0.05$; Table II $)$, suggesting that these rats had developed PSD.

Middle dose E2 improves PSD symptoms. To test if PSD symptoms could be relieved by E2, the present study first determined the dosages of injected E2 to restore the physiological level of E2 in the OVX rats. Females with ovaries exhibit circulating estradiol levels ranging from undetectable to $50 \mathrm{pg} / \mathrm{ml}$. The present study revealed that estradiol, which was injected at 1,10 and $25 \mu \mathrm{g}$ every day, resulted in the low (12.1-14.3 pg/ml), middle (40.3-50.8 pg/ml) and high (100.2-127.5 pg/ml) levels in serum (Table III), suggesting that $10 \mu \mathrm{g}$ E2 administration for 1 or 2 weeks restored physiological E2 levels, which was thus used for PSD treatment. As listed in Tables I and II, PSD symptoms were significantly improved following E2 treatment, based on crossing activity $(\mathrm{P}<0.05)$, rearing frequency $(\mathrm{P}<0.05$; Table $\mathrm{I})$, and
Table II. Sucrose preference intake (\%) in all groups at baseline, at 2 weeks after CMS and at 2 weeks following treatment.

\begin{tabular}{llll}
\hline Group (n) & Baseline & 2W CMS & 2W Treatment \\
\hline CON (16) & $0.74 \pm 0.142$ & $0.65 \pm 0.073$ & $0.64 \pm 0.135$ \\
PSD (16) & $0.78 \pm 0.111$ & $0.35 \pm 0.062^{\mathrm{a}}$ & $0.36 \pm 0.074$ \\
E2 (16) & $0.69 \pm 0.124$ & $0.39 \pm 0.076^{\mathrm{a}}$ & $0.65 \pm 0.082^{\mathrm{b}}$ \\
E2+U0126 (16) & $0.73 \pm 0.076$ & $0.41 \pm 0.072^{\mathrm{a}}$ & $0.48 \pm 0.083$ \\
E2+K252a (16) & $0.72 \pm 0.117$ & $0.33 \pm 0.035^{\mathrm{a}}$ & $0.41 \pm 0.106^{\mathrm{c}}$
\end{tabular}

${ }^{\mathrm{a}} \mathrm{P}<0.05$ vs. $\mathrm{CON} ;{ }^{\mathrm{b}} \mathrm{P}<0.05$ vs. $\mathrm{PSD} ;{ }^{\mathrm{c}} \mathrm{P}<0.05$ vs. E2. CON , control; $\mathrm{PSD}$, post-stroke depression; E2, 17 $\beta$-estradiol; W, week; CMS, chronic mild stress.

Table III. Serum levels of $17 \beta$-estradiol detected after injecting 1,10 or $25 \mu \mathrm{g}$ for 1 or 2 weeks.

\begin{tabular}{lrcr}
\hline E2 dose (n) & 0 week & 1 week & 2 weeks \\
\hline $0 \mu \mathrm{g}(8)$ & $7.6 \pm 2.1$ & $8.3 \pm 3.0$ & $6.3 \pm 1.9$ \\
$1 \mu \mathrm{g}(8)$ & $12.1 \pm 2.3$ & $14.3 \pm 3.1$ & $12.1 \pm 2.9$ \\
$10 \mu \mathrm{g}(8)$ & $11.2 \pm 3.8$ & $40.3 \pm 7.2$ & $50.8 \pm 6.9$ \\
$25 \mu \mathrm{g}(8)$ & $12.5 \pm 2.5$ & $100.2 \pm 15.6$ & $143.5 \pm 20.8$
\end{tabular}

Values report mean serum levels of estradiol pg/ml \pm SD.E2,17ß-estradiol.

SP $(\mathrm{P}<0.05$; Table II $)$, in comparison with vehicle-treated group.

Effects of U0126 and K252a on the behavior tests of PSD rats. To determine the degree at which $\mathrm{E} 2$ effect is mediated by TrkB and MEK-mediated signaling, PSD rats were treated with E2 alone, or E2 combined with SVZ infusions of K252a or U0126, the inhibitors of TrkB and MAPK (MEK), respectively. The present study revealed that PSD rats treated with E2 and K252a or E2 and U0126 exhibited a decrease in frequency of crossing activity and rearing activity compared with the group treated with E2 only $(\mathrm{P}<0.05$; Table I). PSD rats treated with E2 and K252a also showed a significant reduction in SP 

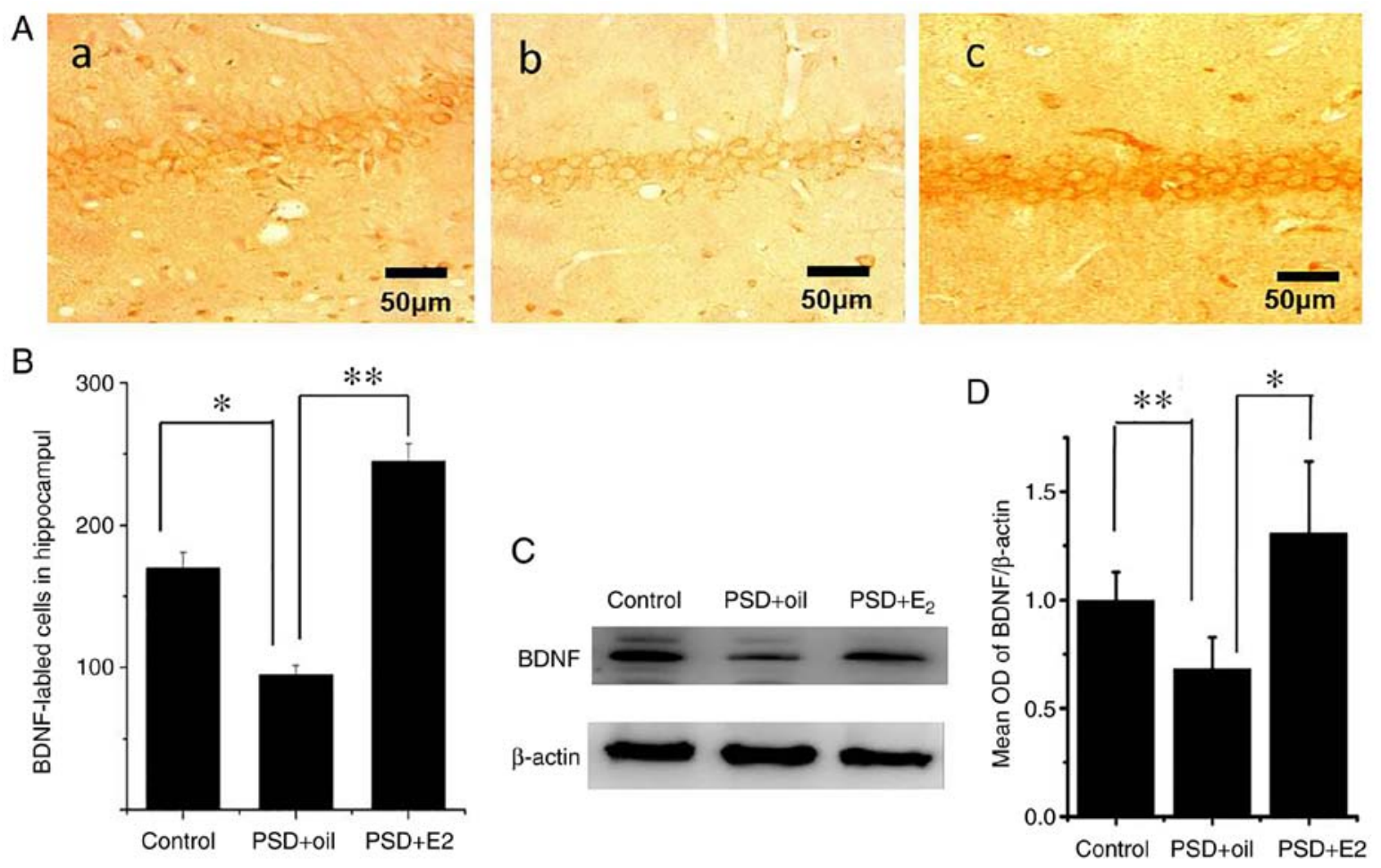

Figure 2. Effects of estrogen treatment on BDNF expression in the hippocampus of rats with PSD. (A) Immunolabeling of BDNF in the hippocampal CA1 region. (Aa) BDNF-positive cells in control group. (Ab) BDNF-positive cells in PSD group. (Ac) BDNF-positive cells in E2 group. (B) Quantification of BDNF cells in the hippocampal CA1 region in the control, PSD and E2 groups. (C) Western blot analysis of BDNF and $\beta$-actin in the control, PSD and E2 groups. (D) Quantitative analysis of western blot images. Data are presented as the mean \pm SEM ( $\mathrm{n}=6$ animals). ${ }^{*} \mathrm{P}<0.05$; ${ }^{* *} \mathrm{P}<0.01$. PSD, post-stroke depression; BDNF, brain-derived neurotrophic factor; E2, 17ß-estradiol; MCAO, middle cerebral artery occlusion; OD, optical density.

compared with E2 treatment alone $(\mathrm{P}<0.05$; Table II). The E2 and U0126 treated group also exhibited a decrease in SP compared with the E2 treated group, even though the decrease was not statistically significant $(\mathrm{P}>0.05$; Table II).

Effects of estrogen therapy on BDNF expression in PSD rats. The present study performed western blot analysis and immunocytochemistry to determine the BDNF expression in the hippocampus of PSD rats following E2 treatment. As presented in Fig. 2, BDNF was detected in the cytoplasm and nuclei of hippocampal cells (Fig. 2Aa-Ac). Immunocytochemistry indicated that the number of BDNF positive cells was significantly lower in the hippocampus CA1 region of $\mathrm{PSD}$ rats $(\mathrm{P}<0.05$ Fig. $2 \mathrm{~B})$, which significantly increased following E2 administration $(\mathrm{P}<0.01$; Fig. 2B). Western blot analysis revealed a significant decrease in BDNF protein expression levels in the hippocampus of PSD rats $(\mathrm{P}<0.01$; Fig. $2 \mathrm{C}$ and $\mathrm{D})$, which was ameliorated following E2 administration $(\mathrm{P}<0.05$; Fig. $2 \mathrm{C}$ and $\mathrm{D})$.

Effects of estrogen therapy on the ratio $p C R E B / C R E B$ and $p \operatorname{TrkB} / \operatorname{TrkB}$ in $P S D$ rats. The present study assessed whether CREB and TrkB activity were affected in PSD rats and by subsequent $\mathrm{E} 2$ treatment. Immunolabeling indicated that pCREB and CREB proteins were expressed in the nuclei of hippocampal dentategyrus region cells (Fig. 3; $\mathrm{Aa}$, control group; Ab, PSD group; Ac, E2 group; and 3B). Immunocytochemistry indicated that the number of pCREB positive cells was significantly decreased in the hippocampus dentategyru region of $\mathrm{PSD}$ rats $(\mathrm{P}<0.05$; Fig. $3 \mathrm{E})$, which significantly improved following $\mathrm{E} 2$ administration $(\mathrm{P}<0.05$; Fig. 3E). Western blot analysis revealed that the pCREB/CREB ratio was significantly decreased in the hippocampus of PSD rats compared with control rats $(\mathrm{P}<0.01$; Fig. $3 \mathrm{C}$ and $\mathrm{F})$. E2 treatment restored the $\mathrm{pCREB} / \mathrm{CREB}$ ratio compared with the untreated PSD group $(\mathrm{P}<0.05 ;$ Fig. $3 \mathrm{C}$ and F). Consistently, the ratio of $\mathrm{pTrkB} / \mathrm{TrkB}$ was decreased in the PSD hippocampus compared with the control group $(\mathrm{P}<0.01$; Fig. $3 \mathrm{D}$ and $\mathrm{G})$, and $\mathrm{E} 2$ treatment increased the ratio of $\mathrm{pTrkB} / \operatorname{TrkB}(\mathrm{P}<0.05$; Fig. 3D and G). These data demonstrated that the CREB activity and TrkB signaling are decreased in PSD rats, which can be reversed by $\mathrm{E} 2$ administration.

Effects of $k 252 a$ therapy on BDNF and pTrkB expression in $P S D$ rats. Since TrkB signaling is able to activate CREB, which in turn activates BDNF, the present study assessed whether the elevation of TrkB signaling by E2 administration could explain the elevation of BDNF expression levels. To test this, the present study injected $\mathrm{k} 252 \mathrm{a}$, the TrkB inhibitor, into SVZ to inhibit TrkB signaling. The results revealed that K252a infusion causes a decrease in total TrkB and pTrkB in comparison with the infusion of aCSF ( $\mathrm{P}<0.01 ;$ Fig. $4 \mathrm{~A}$ and $\mathrm{B})$. Notably, the increase of BDNF expression following E2 administration in PSD rats was nearly completely blocked by the presence of $\mathrm{K} 252 \mathrm{a}(\mathrm{P}<0.01$; Fig. $4 \mathrm{C}$ and $\mathrm{D})$, suggesting that E2 indeed acts through enhancing TrkB signaling to promote BDNF expression.

Effects of U0126 therapy on BDNF and pCREB expression in PSD rats. To assess the mechanism of E2, the 
A a

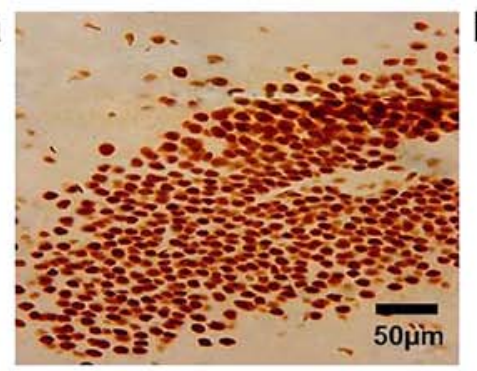

b

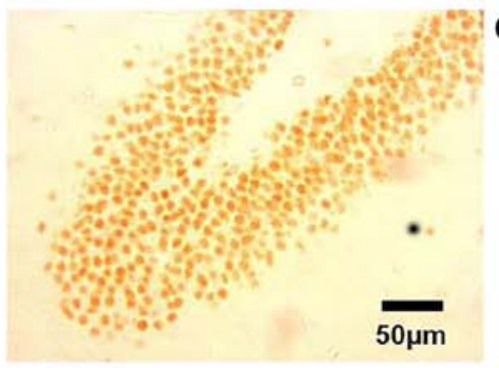

C

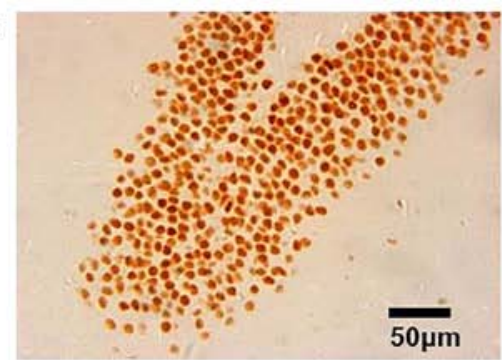

B

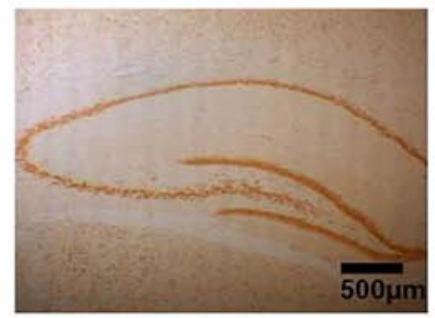

E

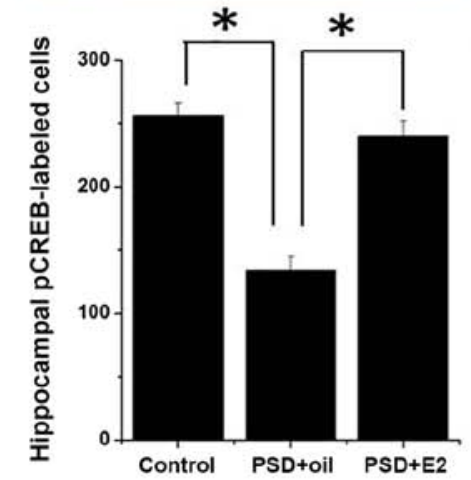

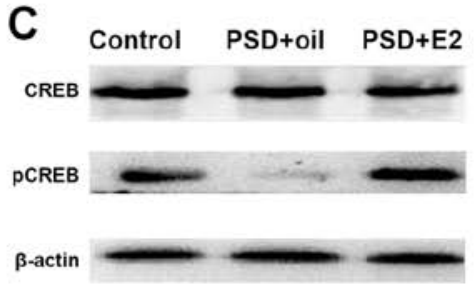

F

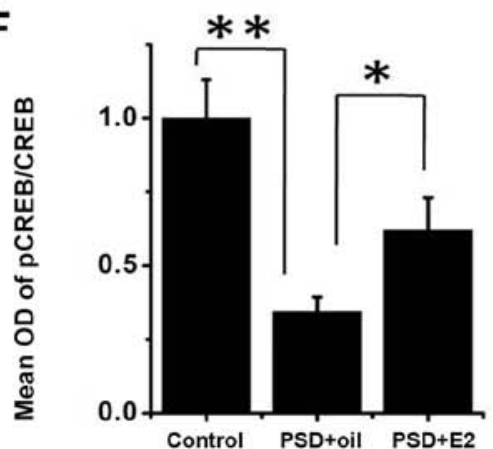

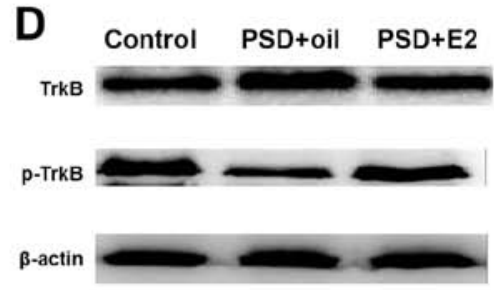

G

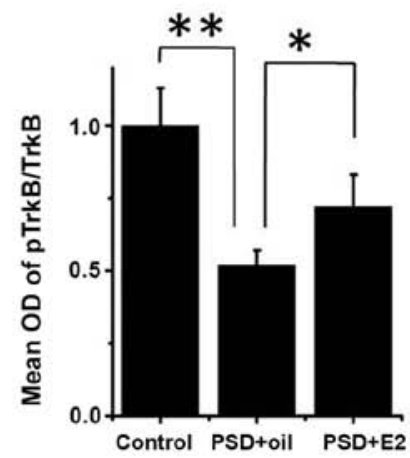

Figure 3. Effects of estrogen treatment on pCREB/CREB and pTrkB/TrkB expression in the hippocampus of PSD rats. (A) Immunolabeling for pCREB in the hippocampal DG region. (Aa) Control group; (Ab) PSD group; (Ac) E2 group. (B) Immunolabeling for CREB in hippocampus. (C) Western blot analysis detected the expected size protein band of pCREB and CREB. (D) Western blot analysis for pTrkB and TrkB in hippocampus in different groups. (E) Quantification of pCREB cells in the hippocampal DG region. (F) Quantitative analysis of western blot images shown in (C). (G) Quantitative analysis of western blot images presented in (D). Data are presented as the mean \pm SEM ( $\mathrm{n}=6$ animals). ${ }^{*} \mathrm{P}<0.05 ;{ }^{* *} \mathrm{P}<0.01$. CREB, cAMP response element-binding protein; PSD, post-stroke depression; DG, dentategyru; p, phosphorylated; E2, 17 $\beta$-estradiol; TrkB, tyrosine kinase B; OD, optical density.

present study examined the role of MEK by injecting the MEK inhibitor U0126 into the SVZ. Western blot analyses showed that the ratio of pCREB/CREB decreased by $34 \%$ following U0126 administration, compared with the aCSF group ( $\mathrm{P}<0.05$; Fig. $5 \mathrm{~A}$ and $\mathrm{B})$, suggesting that $\mathrm{CREB}$ activation in the hippocampus is partially regulated by MEK. Finally, it was revealed that in rats with PSD, the increase in BDNF expression following E2 treatment, indicated by the $\mathrm{BDNF} / \beta$-actin ratio, was attenuated, but not fully blocked by cotreatment of E2 and U0126. The E2 + U0126 group significantly increased the expression of BDNF compared with the oil + U0126 group ( $\mathrm{P}<0.05$; Fig. 5C and $\mathrm{D})$, suggesting that the $\mathrm{E} 2$ effect on BDNF expression is partially dependent on MEK signaling.

\section{Discussion}

At present, there are few studies on the correlation between estrogen and PSD. Previous studies have found that estrogen can significantly improve the depressive behaviors of PSD rats (24), but the mechanism is still unclear. The study established a PSD model in rats by left MCAO followed by exposure to CMS for 2 weeks. It was then revealed that PSD symptoms can be relieved through E2 administration through CREB/BDNF/TrkB signaling.

Depression is a heterogeneous, multifaceted disorder with psychological and behavioral components (8). Translating the complexities of human affective disease (for example, guilt/suicidal symptoms) or of behavioral interventions, such as psychotherapy, into animal models poses a tremendous challenge to the experimental researcher. The present study revealed that a putative PSD model can be developed in rats using MCAO followed by CMS. Rats were subjected to MCAO, which resulted in cerebral infarction that is similar to the pathophysiological mechanisms observed in patients that have experienced a stroke. CMS has been reported to provide an effective equivalent to the precipitation of depression by chronic, low-grade stressors in humans, inducing an anhedonic-like state in rats (32). Both variability and unpredictability during the stress regime are pivotal triggers in the induction of depressive-like behaviors (33). The present study used various behavioral tests to induce depression-like symptoms in rats. SP is regarded to be a key indicator of depression symptoms such as anhedonia, which indicates loss of interest or pleasure $(34,35)$. The frequency of line crossing and rearing activity in the open-field test subjects demonstrated the rats' 

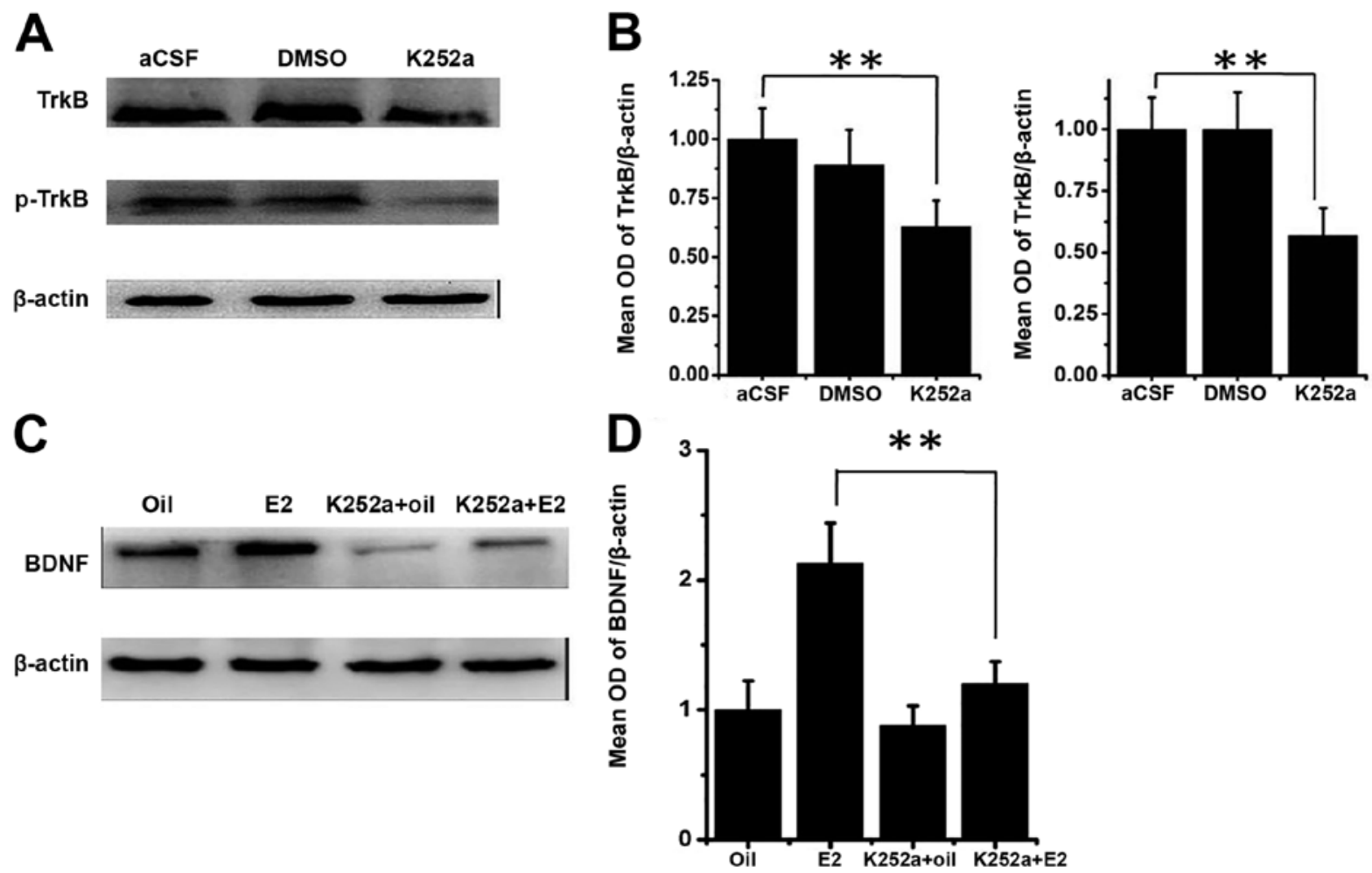

Figure 4. Effects of estrogen administration on subventricle zone infusions of the TrkB inhibitor K252a. (A) Protein levels after infusions of K252a, aCSF and vehicle (DMSO), respectively. (B) Densitometric analysis of western blots shown in (A). (C) Western blot analysis of BDNF and $\beta$-actin in the hippocampus in the K252a, aCSF and vehicle (DMSO) groups. (D) Quantitative analysis for western blotting shown in $(C)$. Values are mean $\pm \mathrm{SEM}(\mathrm{n}=6)$. ${ }^{* *} \mathrm{P}<0.01$. aCSF, artificial cerebrospinal fluid; BDNF, brain-derived neurotrophic factor; $p$, phosphorylated.

locomotor activity and desire to explore. The experimental data in the present study demonstrated that MCAO/CMS rats demonstrated marked performance deficits in both SP index and the open-field test, suggesting the development of PSD symptoms.

The present study also revealed that the depression symptoms in MCAO/CMS rats could largely be reversed following E2 administration. Estrogen has a wide range of effects in the body and brain, and its therapeutic potential for mood, among other physiological and psychological processes, has been recognized for some time (36). E2 administration to young women with low E2 levels may alter mood (37). In women with postpartum depression, administration of sublingual or transdermal E2 sufficiently improves depressive symptoms to meet the definition of clinical recovery (38). E2 also can affect anxiety and depression behavior in animal models. Ovariectomy, which equates to the removal of the primary source of E2, is utilized as a model of E2 deprivation to assess the behavioral effects of E2 (39). OVX rats exhibit increased anxiety and depression behavior, and subcutaneous administration of E2 can reverse these effects $(40,41)$. The experimental data in the present study also indicated that E2 has a robust effect on improving anxiety and depression behavior in rat PSD models.

Mechanistically, estrogen acts through two ERs (ER- $\alpha$ and ER- $\beta$ ) to regulate multiple functions in the central nervous system (42). A previous study on rodent brains have revealed that ER- $\alpha$ is the predominant ER in the hypothalamus, and controls reproduction (43). ER- $\beta$ influences non-reproductive processes and seems to be the main ER subtype expressed in the cerebral cortex, hippocampus, cerebellum and dorsal raphe (44). Estrogen binds to estrogen receptors, acting on MAPK, or directly activating tyrosine kinase, which leads to transcription factors after CREB phosphorylation (19). This further strengthens the cAMP response element-mediated downstream target genes of BDNF transcription, and BDNF then serves an anti-depression role by activating specific receptors, such as TrkB $(19,45,46)$. Phosphorylated TrkB then activates a variety of proteins and enzymes through the cytoplasmic pathway, allowing signals to pass from the cytoplasm into the nucleus, which finally leads to changes in gene expression patterns, affecting the proliferation of neural stem cells, and thus serving a role in regulating depression (47). The present study revealed that in rats with PSD, the activated levels of pTrkB and pCREB were all decreased, which may lead to decreased levels of BDNF. E2 was indicated to enhance TrkB signaling, and was accordingly able to elevate activated CREB and BDNF expression levels. When TrkB signaling was concurrently blocked by k252a, E2 was not able to elevate BDNF expression or to relieve depression symptoms in MCAO/CMS rats. The results of the present study further suggest that this E2 effect is partially dependent on MEK signaling.

The present study consolidated the neurotrophic hypothesis in the development of psychological disorders. BDNF is the key factor of neuronal plasticity, and is closely associated with major depression (48). Antidepressants and the mood stabilizer lithium have both been demonstrated to increase BDNF levels in the brain (49). A previous study reported that a region-specific knockdown of BDNF in the dentate gyrus 
A

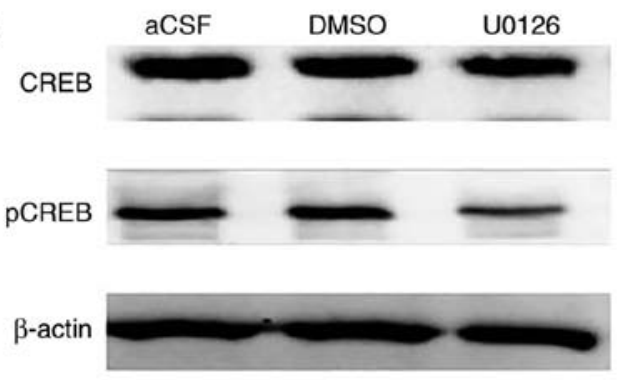

C

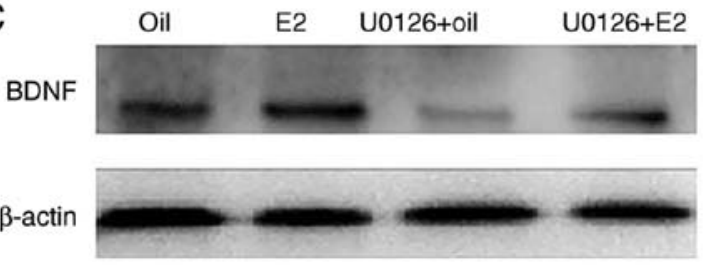

B

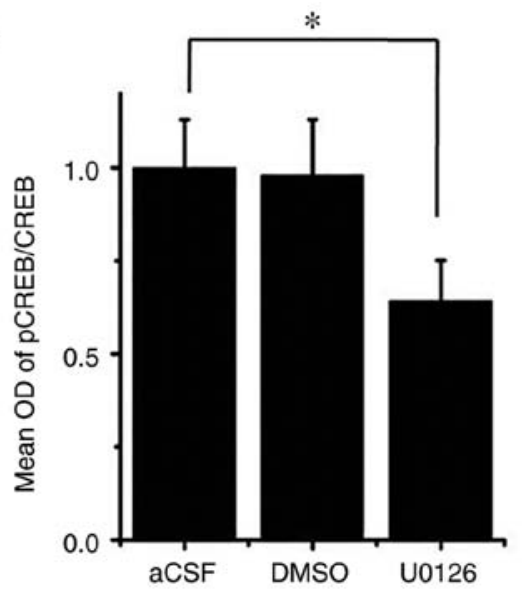

D

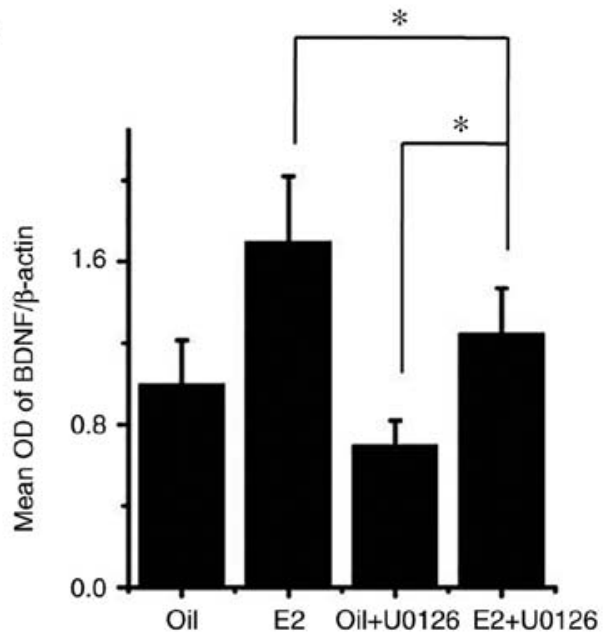

Figure 5. Effects of estrogen treatment on subventricle zone infusions of the MAPK kinase (MEK) inhibitor U0126 on pCREB. (A) Protein levels after U0126, aCSF or vehicle (DMSO) treatment, respectively. (B) Densitometric analysis of western blot analysis shown in (A). (C) Western blot analysis of BDNF and $\beta$-actin in hippocampus in K252a, aCSF and vehicle (DMSO) groups. (D) Quantitative analysis of western blot analysis. Values are presented as the mean \pm SEM $(n=6)$. $P<0.05$. CREB, cAMP response element-binding protein; aCSF, artificial cerebrospinal fluid; BDNF, brain-derived neurotrophic factor; p, phosphorylated; OD, optical density.

induces depression-like behavior (50). Conversely, increased TrkB signaling produced by overexpression of TrkB decreases anxiety and depressive-like behavior $(51,52)$. In patients with PSD, serum concentrations of BDNF decrease (12), and the decreased BDNF-TrkB signaling may therefore serve an important role in the pathogenesis of the illness (53). This coincides with the results observed in the PSD rat model developed in the present study.

Finally, the results of the present study provide novel evidence for a functional interaction between $\mathrm{CREB} / \mathrm{BDNF} / \mathrm{TrkB}$ signaling and estrogen receptor signaling in the hippocampus. In neurons, CREB can be activated by TrkB signaling through phosphorylation, and pCREB will then activate BDNF to enhance TrkB signaling (45). It has been revealed that there is complementary expression of estrogen receptors and BDNF in the hippocampus, and there are similar effects of estrogen and BDNF on hippocampal physiology and behavior (54-56). Previous studies have demonstrated that $\mathrm{E} 2$ administration in OVX rats increases BDNF mRNA and protein expression levels in the hippocampus $(57,58)$, and the mRNA and protein expression of BDNF fluctuate across the estrous cycle in female rats, with the highest levels of BDNF mRNA detected during late diestrus when estrogen reaches the highest levels $(27,59)$. The present study demonstrated that in rats with PSD, E2 treatment is able to elevate CREB/BDNF/TrkB signaling, which in turn leads to a decrease in depression-like symptoms.

In conclusion, the present study developed a PSD model in rats, whose depression-like symptoms can be relieved by estrogen treatment that acts to promote CREB/BDNF/TrkB signaling. Considering that serum BDNF levels were reported to be decreased in human patients with PSD, future studies should focus on whether estrogen treatment could be a therapeutic agent that can be used to treat these patients.

\section{Acknowledgements}

Not applicable.

\section{Funding}

The current study was supported by grants from Wenzhou Technology Foundation (grant no. H20090067) and the National Institute of Health (nos. AG21980 and NS057186). 


\section{Availability of data and materials}

All data generated or analyzed during this study are included in this published article.

\section{Authors' contributions}

HJ, LX, KJ and BS designed the study and performed the experiments. HJ and LX established the animal models, collected the data and analyzed the data. HJ wrote the manuscript. BS revised the manuscript. All authors read and approved the final version of the manuscript.

\section{Ethics approval and consent to participate}

The present study was approved by the Animal Ethics Committee of First Affiliated Hospital, Wenzhou Medical University (Wenzhou, China).

\section{Patient consent for publication}

Not applicable.

\section{Competing interests}

The authors declare they have no competing interests.

\section{References}

1. Kim AS and Johnston SC: Temporal and geographic trends in the global stroke epidemic. Stroke 44 (Suppl 1): S123-S125, 2013

2. Liu M, Wu B, Wang WZ, Lee LM, Zhang SH and Kong LZ: Stroke in China: Epidemiology, prevention, and management strategies. Lancet Neurol 6: 456-464, 2007.

3. Boden-Albala B, Litwak E, Elkind MS, Rundek T and Sacco RL: Social isolation and outcomes post stroke. Neurology 64: 1888-1892, 2005.

4. Ellis C, Zhao Y and Egede LE: Depression and increased risk of death in adults with stroke. J Psychosomatic Res 68: 545-551, 2010.

5. Hackett ML, Yapa C, Parag V and Anderson CS: Frequency of depression after stroke: A systematic review of observational studies. Stroke 36: 1330-1340, 2005.

6. Poynter B, Shuman M, Diaz-Granados N, Kapral M, Grace SL and Stewart DE: Sex differences in the prevalence of post-stroke depression: A systematic review. Psychosomatics 50: 563-569, 2009.

7. Hackett ML and Pickles K: Part I: Frequency of depression after stroke: An updated systematic review and meta-analysis of observational studies. Int J Stroke 9: 1017-1025, 2014.

8. Loubinoux I, Kronenberg G, Endres M, Schumann-Bard P, Freret T, Filipkowski RK, Kaczmarek L and Popa-Wagner A: Post-stroke depression: Mechanisms, translation and therapy. J Cell Mol Med 16: 1961-1969, 2012.

9. Duman RS and Monteggia LM: A neurotrophic model for stress-related mood disorders. Biol Psychiatry 59: 1116-1127, 2006.

10. McEwen BS: Protective and damaging effects of stress mediators: Central role of the brain. Dialogues Clin Neurosci 8: 367-381, 2006.

11. Krishnan V and Nestler EJ: The molecular neurobiology of depression. Nature 455: 894-902, 2008.

12. Yang L, Zhang Z, Sun D, Xu Z, Yuan Y, Zhang X and Li L: Low serum BDNF may indicate the development of PSD in patients with acute ischemic stroke. Int J Geriatric Psychiatry 26 : 495-502, 2011

13. Pillai A: Brain-derived neurotropic factor/TrkB signaling in the pathogenesis and novel pharmacotherapy of schizophrenia. Neurosignals 16: 183-193, 2008.
14. Horch HW and Katz LC: BDNF release from single cells elicits local dendritic growth in nearby neurons. Nat Neurosci 5: 1177-1184, 2002.

15. Bergami M, Rimondini R, Santi S, Blum R, Götz M and Canossa M: Deletion of TrkB in adult progenitors alters newborn neuron integration into hippocampal circuits and increases anxiety-like behavior. Proc Natil Acad Sci 105: 15570-15575, 2008.

16. Sanchez-Huertas C and Rico B: CREB-Dependent regulation of GAD65 transcription by BDNF/TrkB in cortical interneurons. Cereb Cortex 21: 777-788, 2011.

17. Blendy JA: The role of CREB in depression and antidepressant treatment. Biol Psychiatry 59: 1144-1150, 2006.

18. Nibuya M, Nestler EJ and Duman RS: Chronic antidepressant administration increases the expression of cAMP response element binding protein (CREB) in rat hippocampus. J Neurosci 16: 2365-2372, 1996.

19. Carlezon WA Jr, Duman RS and Nestler EJ: The many faces of CREB. Trends Neurosci 28: 436-445, 2005.

20. Romano-Torres M and Fernández-Guasti A: Estradiol valerate elicits antidepressant-like effects in middle-aged female rats under chronic mild stress. Behav Pharmacol 21: 104-111, 2010.

21. Lebesgue D, Traub M, De Butte-Smith M, Chen C, Zukin RZ, Kelly MJ and Etgen AM: Acute administration of non-classical estrogen receptor agonists attenuates ischemia-induced hippocampal neuron loss in middle-aged female rats. PLoS One 5: e8642, 2010.

22. Ormerod BK, Lee TT and Galea LA: Estradiol enhances neurogenesis in the dentate gyri of adult male meadow voles by increasing the survival of young granule neurons. Neuroscience 128: 645-654, 2004

23. Scharfman HE and MacLusky NJ: Similarities between actions of estrogen and BDNF in the hippocampus: Coincidence or clue? Trends Neurosci 28: 79-85, 2005.

24. Su Q, Cheng Y, Jin K, Cheng J, Lin Y, Lin Z, Wang L and Shao B: Estrogen therapy increases BDNF expression and improves post-stroke depression in ovariectomy-treated rats. Exp Ther Med 12: 1843-1848, 2016.

25. Kight KE and McCarthy MM: Sex differences and estrogen regulation of BDNF gene expression, but not propeptide content, in the developing hippocampus. J Neurosci Res 95: 345-354, 2017.

26. Yang NB, Pan XJ, Cheng JJ, Lin JQ and Zhu JY: Ethical inspection about laboratory animals. Zhongguo Ying Yong Sheng Li Xue Za Zhi 31: 504-507, 2015.

27. Allen AL and McCarson KE: Estrogen increases nociception-evoked brain-derived neurotrophic factor gene expression in the female rat. Neuroendocrinology 81: 193-199, 2005.

28. Gao BY, Sun CC, Xia GH, Zhou ST, Zhang Y, Mao YR, Liu PL, Zheng Y, Zhao D, Li XT, et al: Paired associated magnetic stimulation promotes neural repair in the rat middle cerebral artery occlusion model of stroke. Neural Regen Res 15: 2047-2056, 2020.

29. Li X, Chen C, Yang X, Wang J, Zhao ML, Sun H, Zhang S and Tu Y: Acupuncture improved neurological recovery after traumatic brain injury by activating BDNF/TrkB pathway. Evid Based Complement Alternat Med 2017: 8460145, 2017.

30. Wang ZQ, Chen XC, Yang GY and Zhou LF: U0126 prevents erk pathway phosphorylation and interleukin-1 $\beta$ mRNA prodution after cerebral ischemia. Chin Med Sci J 19: 270-275, 2004.

31. Yi LT,Li J, Liu BB, Luo L, Liu Q and Geng D: BDNF-ERK-CREB signalling mediates the role of miR-132 in the regulation of the effects of oleanolic acid in male mice. J Psychiatry Neurosci 39: 348-359, 2014.

32. Willner P: Chronic mild stress (CMS) revisited: Consistency and behavioural-neurobiological concordance in the effects of CMS. Neuropsychobiology 52: 90-110, 2005.

33. Chang $\mathrm{CH}$ and Grace AA: Amygdala-Ventral pallidum pathway decreases dopamine activity after chronic mild stress in rats. Biol Psychiatry 76: 223-230, 2014.

34. Kalueff AV, Gallagher PS and Murphy DL: Are serotonin transporter knockout mice'depressed'?: Hypoactivity but no anhedonia. Neuroreport 17: 1347-1351, 2006.

35. Strekalova T, Gorenkova N, Schunk E, Dolgov O and Bartsch D: Selective effects of citalopram in a mouse model of stress-induced anhedonia with a control for chronic stress. Behav Pharmacol 17: 271-287, 2006

36. Newhouse $P$ and Albert K: Strogen, stress, and depression: A neurocognitive model. JAMA Psychiatry 72: 727-729, 2015.

37. Schmidt PJ: Depression, the perimenopause, and estrogen therapy. Ann NY Acad Sci 1052: 27-40, 2005. 
38. Ahokas A, Kaukoranta J, Wahlbeck K and Aito M: Estrogen deficiency in severe postpartum depression: Successful treatment with sublingual physiologic 17beta-estradiol: A preliminary study. J Clin Psychiatry 62: 332, 2001.

39. Cheng Y, Su Q, Shao B, Cheng J, Wang H, Wang L, Lin Z, Ruan L, ZhuGe Q and Jin K: 17 $\beta$-Estradiol attenuates poststroke depression and increases neurogenesis in female ovariectomized rats. BioMed Res Int 2013: 1-10, 2013.

40. Bowman R, Ferguson D and Luine VN: Effects of chronic restraint stress and estradiol on open field activity, spatial memory, and monoaminergic neurotransmitters in ovariectomized rats. Neuroscience 113: 401-410, 2002.

41. Walf AA and Frye CA: Estradiol's effects to reduce anxiety and depressive behavior may be mediated by estradiol dose and restraint stress. Neuropsychopharmacology 30: 1288-1301, 2005.

42. Mitra SW, Hoskin E, Yudkovitz J, Pear L, Wilkinson HA Hayashi S, Pfaff DW, Ogawa S, Rohrer SP, Schaeffer JM, et al: Immunolocalization of estrogen receptor beta in the mouse brain: Comparison with estrogen receptor alpha. Endocrinology 144 2055-2067, 2003.

43. Lorsch ZS, Loh YE, Purushothaman I, Walker DM, Parise EM, Salery M, Cahill ME, Hodes GE, Pfau ML, Kronman H, et al: Estrogen receptor alpha drives pro-resilient transcription in mouse models of depression. Nat Commun 9: 1116, 2018.

44. Suzuki S, Gerhold LM, Böttner M, Rau SW, Cruz CD, Yang E, $\mathrm{Zhu} \mathrm{H}$, Yu J, Cashion AB, Kindy MS, et al: Estradiol enhances neurogenesis following ischemic stroke through estrogen receptors alpha and beta. J Comp Neurol 500: 1064-1075, 2007.

45. Nair A, Vadodaria KC, Banerjee SB, Benekareddy M, Dias BG Duman RS and Vaidya VA: Stressor-Specific regulation of distinct brain-derived neurotrophic factor transcripts and cyclic AMP response element-binding protein expression in the postnatal and adult rat hippocampus. Neuropsychopharmacology 32 : $1504-1519,2007$.

46. Wu Q, Chambliss K, Umetani M, Mineo C and Shaul PW: Non-nuclear estrogen receptor signaling in the endothelium. J Biol Chem 286: 14737-14743, 2011.

47. Rantamäki T and Castrén E: Targeting TrkB neurotrophin receptor to treat depression. Expert Opin Ther Targets 12: $705-715,2008$

48. Pezawas L, Meyer-Lindenberg A, Goldman AL, Verchinski BA, Chen G, Kolachana BS, Egan MF, Mattay VS, Hariri AR and Weinberger DR: Evidence of biologic epistasis between BDNF and SLC6A4 and implications for depression. Mol Psychiatry 13 709-716, 2008

49. Yasuda S, Liang M, Marinova Z, Yahyavi A and Chuang D: The mood stabilizers lithium and valproate selectively activate the promoter IV of brain-derived neurotrophic factor in neurons. Mol Psychiatry 14: 51-59, 2007.
50. Taliaz D, Stall N, Dar D and Zangen A: Knockdown of brain-derived neurotrophic factor in specific brain sites precipitates behaviors associated with depression and reduces neurogenesis. Mol Psychiatry 15: 80-92, 2009.

51. Koponen E, Rantamäki T, Voikar V, Saarelainen T, MacDonald E and Castrén E: Enhanced BDNF signaling is associated with an antidepressant-like behavioral response and changes in brain monoamines. Cell Mol Neurobiol 25: 973-980, 2005.

52. Koponen E, Võikar V, Riekki R, Saarelainen T, Rauramaa T, Rauvala H, Taira T and Castrén E: Transgenic mice overexpressing the full-length neurotrophin receptor trkB exhibit increased activation of the trkB-PLC $\gamma$ pathway, reduced anxiety, and facilitated learning. Mol Cell Neurosci 26: 166-181, 2004.

53. Zhang $\mathrm{E}$ and Liao P: Brain-Derived neurotrophic factor and post-stroke depression. J Neurosci Res 89: 537-548, 2020.

54. Scharfman HE and MacLusky NJ: Estrogen and brain-derived neurotrophic factor (BDNF) in hippocampus: Complexity of steroid hormone-growth factor interactions in the adult CNS. Front Neuroendocrinology 27: 415-435, 2006.

55. Scharfman HE, Mercurio TC, Goodman JH, Wilson MA and MacLusky NJ: Hippocampal excitability increases during the estrous cycle in the rat: A potential role for brain-derived neurotrophic factor. J Neurosci 23: 11641-11652, 2003.

56. Harte-Hargrove L, MacLusky NJ and Scharfman HE: Brain-derived neurotrophics factor-estrogen interactions in hippocampal mossy fiber pathway: Implications for normal brain function and disease. Neuroscience 3: 46-66, 2012.

57. Jezierski $M$ and Sohrabji F: Neurotrophin expression in the reproductively senescent forebrain is refractory to estrogen stimulation. Neurobiol Aging 22: 309-319, 2001.

58. Zhou J, Zhang H, Cohen RS and Pandey SC: Effects of estrogen treatment on expression of brain-derived neurotrophic factor and cAMP response element-binding protein expression and phosphorylation in rat amygdaloid and hippocampal structures. Neuroendocrinology 81: 294-310, 2005.

59. Gibbs RB: Levels of trkA and BDNF mRNA, but not NGF mRNA, fluctuate across the estrous cycle and increase in response to acute hormone replacement. Brain Res 787: 259-268, 1998.

This work is licensed under a Creative Commons Attribution-NonCommercial-NoDerivatives 4.0 International (CC BY-NC-ND 4.0) License. 